ARTIGO

ORIGINAL

\title{
Epidemiologia dos acidentes no surfe no Brasil
}

\author{
J. Steinman, E.H. Vasconcellos, R.M. Ramos, J.L. Botelho e M.V. Nahas \\ Nupaf - Centro de Desportos Universidade Federal de Santa Catarina
}

\section{RESUMO}

Apesar do grande número de praticantes do surfe no Brasil, nenhum trabalho científico nacional foi dedicado à caracterização e análise epidemiológica das lesões esportivas desse grupo. Pretendeu-se com este estudo identificar os tipos e lesões mais freqüentes relacionadas à prática do surfe recreacional e competitivo no Brasil. Estudaram-se suas causas, a parte do corpo atingida, sua gravidade, relacionadas às diferentes condições de surfe. Foram distribuídos 21.300 questionários pelos Estados litorâneos do Nordeste, Sudeste e Sul do Brasil. Um banco de dados foi montado com respostas de 930 praticantes, que relataram 927 lesões que necessitaram atenção médica ou que impediram a prática do surfe por um ou mais dias (num período de três anos). A população incluiu $67,5 \%$ surfistas recreacionais, $29,4 \%$ amadores e $3,1 \%$ profissionais, a maior parte do sexo masculino (95,3\%); 56,6\% praticam o esporte há mais de cinco anos. A média de idade foi de 23,7 anos (DP $=6,30)$. A maioria das lesões foi de natureza traumática $(82,5 \%)$ e ocorreu durante a prática do surfe recreacional (96,2\%). As lesões mais comuns foram as lacerações (44\%), as contusões $(16,9 \%)$ e as lesões musculoligamentares (15,5\%); $38 \%$ das lesões atingiram os membros inferiores, $17,9 \%$ os membros superiores e $15,6 \%$ a cabeça. O tempo de afastamento da prática do surfe devido à lesão foi: $54,2 \%$ até 7 dias, $20,7 \%$ entre 7 e 14 dias, $10,1 \%$ entre 14 e 30 dias e $14,8 \%$ mais de 30 dias. Nesta amostra, a incidência de lesões foi de 2,47 para cada mil dias de surfe. Esta incidência foi inferior à obtida em outros estudos. Apesar da alta incidência de lesões traumáticas neste estudo, a alta prevalência de dores lombares recorrentes $(28,4 \%)$, dores no pescoço $(27,3 \%)$, dores nos ombros $(20,5 \%)$ e dores nos joelhos $(12,5 \%)$ sugere que as lesões de esforço repetido sejam um problema comum entre os surfistas.

Palavras-chave: Epidemiologia. Acidentes. Lesões. Surfe.

Endereço para correspondência:

Centro de Acupuntura \& Medicina Esportiva \& Reabilitação Aquática

Waterway

Rua Cruz e Souza, 73 - Centro

88020-710 - Florianopolis, SC

Tel./fax: 228-7000

E-mail: waterway@brasilnet.com.br

Rev Bras Med Esporte - Vol. 6, N 1 - Jan/Fev, 2000

\section{ABSTRACT}

\section{Epidemiology of surfing accidents in Brazil}

Surfing has become extremely popular in Brazil. Information on the rate and spectrum of surfing injuries in Brazil is quite limited. The objectives of this study were to investigate the incidence of surfing injuries in Brazil and to analyze the types, causes, part of body, and severity of injuries associated to recreational and competitive surfing, and related to different surfing conditions. Nine hundred and thirty surfers answered a comprehensive questionnaire and reported 927 injuries (over a period of three years) requiring medical attention or resulting in inability to surf for one or more days. The population included recreational (67.5\%), amateur (29.4\%), and professional (3.1\%) male surfers. Mean age was 23.7 years $(S D=6.30)$. Fiftynine percent had more than 5 years of surfing experience. Most injuries were traumatic in nature (82.5\%) and occurred during free surfing (96.2\%). The most common injuries were laceration (44\%), contusion (16.9\%), muscle and ligament injury (15.5\%). Thirty-eight percent of the injuries were in the lower limbs, $17.9 \%$ in the upper limbs, $15.6 \%$ in the head. Total restriction from surfing following injuries was $54.2 \%$ up to 7 days, 20.7\% from 7 to 14 days, $10.1 \%$ from 14 to 30 days, and $14.8 \%$ more than 30 days. The rate of moderate to severe injuries among this sample was 2.47 injuries per 1000 surfing days. Compared to other studies, the total incidence was low. Despite the high incidence of traumatic injuries, the prevalence of recurrent lower back pain (28.4\%), neck pain (27.3\%), shoulder pain (20.5\%), and knee pain (12.5\%) in this study suggest overuse injuries as a common problem among surfers.

Key words: Epidemiology. Surfing. Accidents. Injuries.

\section{INTRODUÇÃO}

Com o seu litoral atlântico, que se estende de Belém do Pará (latitude próxima a zero) ao Rio Grande do Sul (latitude próxima aos 40 graus sul), com mais de $4.000 \mathrm{~km}$ de extensão e que oferece excelentes condições à prática deste esporte aquático em nível recreacional, o surfe brasileiro tem-se destacado no cenário internacional das competi- 
ções amadoras e profissionais e atualmente ocupa e disputa ponto a ponto a liderança dos títulos mundiais ao lado da Austrália e Estados Unidos. Isso tem sido responsável pelo marcante crescimento da prática do surfe no Brasil, desde a sua introdução no começo dos anos 60. Assessorados pela recém-fundada Confederação Brasileira de Surfe e pela Associação Brasileira de Surfe Profissional (Abrasp), aliado à participação crescente da mídia não especializada (jornais e televisão), a prática do surfe nas praias brasileiras tem atraído cada vez mais crianças e adolescentes. Por ser praticado em ambiente oceânico, algumas vezes de caráter hostil, o indivíduo é exposto a diferentes condições ambientais que incluem: o tempo e suas variantes, como a temperatura, o vento, sol, chuva, o tipo de fundo oceânico, as condições do mar, o tamanho das ondas e as variações da maré. Associa-se, de maneira geral, o surfe a um esporte de alto risco para acidentes, quando comparado com outros esportes, mesmo os ditos "radicais" (skate, windsurfe, pára-quedismo, asa delta, parapente, canoagem, mergulho, roller blade e esqui na neve). Entretanto, ao comparar esportes de risco, a United States Consumer Product Safety Commission, em 1979, relatou enorme diferença entre a incidência de lesões no surfe (9.900) com as lesões no skateboard $(137.900)^{1}$. Das poucas publicações científicas que estudam o tema "acidentes no surfe", merecem destaque os estudos australianos de Lowdon et al. ., $^{2,3}$ que descrevem uma incidência de 3,5 acidentes de média a grave seriedade para cada 1.000 dias de prática do surfe recreacional, contra 4,0 acidentes para cada 1.000 dias de surfe competitivo. Esses estudos colocam o surfe como um esporte aquático relativamente seguro. Apesar do crescimento no número de praticante neste esporte, não existem informações precisas sobre as lesões esportivas nesse grupo, não havendo nenhum estudo científico nacional dedicado ao estudo da epidemiologia das lesões relacionadas à prática do surfe no Brasil. Pretendeu-se determinar a incidência, tipo, gravidade e localização das lesões traumáticas e não-traumáticas agudas e crônicas, relativas à prática do surfe no Brasil e relacioná-las à idade, sexo, grau de experiência, categoria do praticante e condições do mar, além de propor medidas preventivas para evitá-las.

\section{METODOLOGIA}

Foi feito um levantamento epidemiológico retrospectivo, buscando caracterizar e descrever a incidência das lesões traumáticas e não traumáticas, agudas e crônicas, segundo as variáveis epidemiológicas clássicas, considerando o perfil do praticante e condições da prática. O tipo e a localização anatômica da lesão estão de acordo com a classificação internacional da Organização Mundial de Saúde ${ }^{4}$ por nós ampliada. Fizeram parte da amostra os surfistas amadores, profissionais e recreacionais.

De janeiro a setembro de 1997 foram distribuídos 21.300 copias de um questionário do tipo "porte pago", previamente validado, incluindo dados pessoais, características da prática e dados relativos a acidentes/lesões relacionados à prática do surfe. A distribuição foi feita da seguinte maneira:

1) 300 questionários por mala direta das Associações Brasileiras de Surfe Amador e Profissional (Abrasa e Abrasp); 2) mil questionários nas competições esportivas ocorridas durante a época de coleta dos dados; 3 ) oito mil questionários nas principais surf shops do Brasil; 4) 12 mil questionários foram encartados em revista especializada no esporte (Revista Inside), de distribuição nacional.

Visando estimular o maior numero de respostas completas e corretas, uma série de premiações foi sorteada entre aqueles que responderam adequadamente à pesquisa. Tais premiações foram obtidas através de um programa de apoio junto a diferentes empresas envolvidas com o mercado do surfe (confecções, pranchas, roupas de borracha).

Um banco de dados foi montado utilizando-se o software Epi-Info 6.O da Organização Mundial de Saúde. Os dados descritivos foram tabulados e as análises de causalidade foram feitas a partir do cálculo da odds ratio, com intervalo de confiança de $95 \%$, e testadas pela prova do quiquadrado. Para as análises de variáveis quantitativas foram utilizados os testes de correlação e regressão múltipla. A significância foi estabelecida em $\mathrm{p}<0,05$.

\section{RESULTADOS}

Dos 20.000 questionários disponibilizados por todo o litoral brasileiro, 930 foram respondidos correta e completamente. Destes, 679 (73\%) relatam ter sofrido 927 lesões que requereram atendimento médico ou o impediram de surfar por um ou mais de um dia. A maioria dos sujeitos era do sexo masculino $(95,3 \%)$ e a média de idade foi de

\begin{tabular}{crc}
\hline \multicolumn{3}{c}{ TABELA 1} \\
Freqüência de sujeitos que reportaram lesões por faixa \\
etária
\end{tabular}


23,7 anos ( $\mathrm{DP}=6,3)$ As lesões predominaram na faixa etária dos 15 aos $24 \operatorname{anos}(55 \%)$ e dos 25 aos $34 \operatorname{anos}(33,4 \%)$. Como não houve diferença significativa nos resultados entre os sexos, as informações para ambos foram agrupadas (tabela 1).

Mais da metade dos sujeitos $(59,1 \%)$ pratica o surfe há mais de cinco anos e a maioria é de surfistas recreacionais $(69,1 \%)$. Isto caracteriza que a amostra é composta por praticantes com significativa experiência no esporte, o que nos leva a supor a veracidade das informações (tabela 2 ).

Devido à metodologia adotada, que incluiu maior divulgação regional da pesquisa, nos Estados do Sul e Sudeste, a maior parte da amostra provém dos Estados de São Paulo (21\%), seguido de Santa Catarina (22\%) e Rio de Janeiro (6\%). Apesar disso, um número significativo da amostra provém da região Nordeste (44\%).

Das 927 lesões, $82,6 \%$ foram de origem traumática e ocorreram durante o surfe recreacional. As lesões mais comuns foram as lacerações em 44\%, as contusões em 16,9\% e as lesões musculoligamentares em 15,5\% (tabela 3). Apesar da predominância de lesões traumáticas neste estudo,

\begin{tabular}{lrr}
\hline \multicolumn{3}{c}{ TABELA 2} \\
Experiência do praticante \\
\hline Experiência & Lesão & $\%$ \\
& & \\
Menos de 2 anos & 121 & 17,8 \\
2 a 5 anos & 152 & 22,4 \\
Mais de 5 anos & 334 & 49,2 \\
Não responderam & 72 & 10,6 \\
Total & 679 & 100 \\
\hline
\end{tabular}

registramos alta prevalência de dores lombares recorrentes $(28,4 \%)$, dores no pescoço $(27,3 \%)$, dores nos ombros $(20,5 \%)$ e dores nos joelhos $(12,5 \%)$. Além disso, mais da metade dos surfistas estudados, $53 \%$, surfava entre 10 hoo e $15 \mathrm{hoo}$, estando, portanto, mais expostos aos efeitos negativos do sol.

Com uma média de prática de 2,6 dias/semana, 2,1 sessões de surfe por dia, com duração média de 2,68 horas, a incidência de lesões (que necessitaram atenção médica ou que impediram a prática do surfe por um ou mais dias) foi de 2,47 para cada mil dias de surfe. Esta incidência foi inferior à obtida no estudo de Lowdon et al. ${ }^{2}$ com surfistas australianos.

\begin{tabular}{lrr}
\hline \multicolumn{3}{c}{$\begin{array}{c}\text { TABELA 3 } \\
\text { Incidência e tipo de lesão }\end{array}$} \\
\multicolumn{1}{c}{ Incidência } & N & \multicolumn{1}{c}{$\%$} \\
Laceração & 408 & \multicolumn{1}{c}{44} \\
Contusão & 157 & 16,9 \\
Estiramento músculo/ligamentar & 144 & 15,5 \\
Fratura & 23 & 2,5 \\
Luxação & 30 & 3,2 \\
Perf. tímpano & 5 & 0,5 \\
Queimadura & 83 & 8,9 \\
Infec. ouvido & 31 & 3,5 \\
Gastroenterite aguda & 2 & 0,3 \\
Hipotermia & 3 & 0,3 \\
Hérnia de disco & 7 & 0,8 \\
Afogamento & 7 & 0,8 \\
Outros & 25 & 2,8 \\
Total & 927 & 100 \\
\hline
\end{tabular}

\begin{tabular}{|c|c|c|c|c|c|c|c|c|}
\hline \multicolumn{9}{|c|}{$\begin{array}{c}\text { TABELA } 4 \\
\text { Região do corpo lesionada por tipo de lesão - n (\%) }\end{array}$} \\
\hline $\begin{array}{l}\text { Localização } \\
\text { anatômica }\end{array}$ & & $\begin{array}{l}\text { lbeça e } \\
\text { escoço }\end{array}$ & Tronco & $\begin{array}{l}\text { Membros } \\
\text { superiores }\end{array}$ & $\begin{array}{l}\text { Membros } \\
\text { inferiores }\end{array}$ & Indefinida & & Total \\
\hline \multicolumn{9}{|l|}{ Tipo } \\
\hline Corte & 90 & $(17,5 \%)$ & $17(3,3 \%)$ & $80(15,6 \%)$ & $189(36,9 \%)$ & $137(26,7 \%)$ & 513 & $(100 \%)$ \\
\hline Contusão & 46 & $(29,5 \%)$ & $12(7,7 \%)$ & $18(11,6 \%)$ & $52(33,3 \%)$ & $28(17,9 \%)$ & 156 & $(100 \%)$ \\
\hline Distensão muscular & & $(3,3 \%)$ & $30(32,3 \%)$ & $18(19,3 \%)$ & $42\left(45,1 \frac{1}{\circ}\right)$ & - & 93 & $(100 \%)$ \\
\hline Entorse ligamentar & & - & $2(3,4 \%)$ & $6(10,2 \%)$ & $42\left(71,2 \frac{\circ}{\circ}\right)$ & $9\left(15,2 \frac{\circ}{0}\right)$ & 59 & $(100 \%)$ \\
\hline Fratura & & $(9,6 \%)$ & $4(17,4 \%)$ & $4(17,4 \%)$ & $13(56,6 \%)$ & - & 23 & $(100 \%)$ \\
\hline Luxação & & - & - & $13(43,3 \%)$ & $9(30 \%)$ & $8\left(26,7 \frac{\circ}{\circ}\right)$ & 30 & $(100 \%)$ \\
\hline Hérnia de disco & & - & $7(100 \%)$ & - & - & - & 7 & $(100 \%)$ \\
\hline $\begin{array}{l}\text { Queimadura } \\
\text { por água viva }\end{array}$ & & $(9,2 \%)$ & $8(9,2 \%)$ & $35(40,3 \%)$ & $19(21,8 \%)$ & $17(19,5 \%)$ & 87 & $(100 \%)$ \\
\hline Total geral & 149 & $(15,6 \%)$ & $80(8,2 \%)$ & $174(17,9 \%)$ & $366(38 \%)$ & $199(20,5 \%)$ & 968 & $(100 \%)$ \\
\hline
\end{tabular}


As diversas partes do corpo foram lesionadas 968 vezes, sendo que $38 \%$ das lesões atingiram os membros inferiores, $17,9 \%$ os membros superiores e $15,6 \%$ a cabeça. Os cortes $(44 \%)$ foram mais freqüentes nos membros inferiores, sendo os pés (21,9\%) pernas(10,8\%) os mais lesionados. A região da cabeça foi atingida em 21,9\%, sendo o crânio $(9,6 \%)$ e o rosto $(4,8 \%)$ os mais acometidos. Nos membros superiores, $19,2 \%$ dos cortes atingiram principalmente o braço $(4,8 \%)$ e a mão $(2,1 \%)$ (tabela 4 ).

As contusões (16,9\%) atingiram o membro inferior em $31,81 \%$, sendo os joelhos (11\%) e pernas (11\%) igualmente os mais acometidos. A região da cabeça foi atingida em $30,5 \%$, sendo o crânio (14,2\%), o nariz $(4,5 \%)$ e o rosto $(3,2 \%)$ os mais lesionados.

As distensões musculares (9,2\%) atingiram o membro inferior em $45 \%$, em particular a região da coxa $(21,4 \%) \mathrm{e}$

\begin{tabular}{|c|c|}
\hline \multicolumn{2}{|c|}{$\begin{array}{c}\text { TABELA } 5 \\
\text { Causas imediatas das lesões }\end{array}$} \\
\hline Causa da lesão & $\%$ \\
\hline Prancha & 35,9 \\
\hline Manobras & 35,1 \\
\hline Outros & 29,0 \\
\hline \multicolumn{2}{|c|}{$\begin{array}{c}\text { TABELA } 6 \\
\text { Parte da prancha envolvida na lesão }\end{array}$} \\
\hline \multicolumn{2}{|c|}{ Parte da prancha envolvida na lesão } \\
\hline Parte da prancha & $\%$ \\
\hline $\mathrm{Bico}$ & 11,9 \\
\hline Rabeta & 10,1 \\
\hline Quilha & 55,2 \\
\hline Cordinha & 2,7 \\
\hline Não definido/outras & 20,1 \\
\hline \multicolumn{2}{|c|}{ TABELA 7} \\
\hline Manobras de surfe envol & cidentes \\
\hline Manobra & $\%$ \\
\hline Drop & 15,5 \\
\hline Botton turn & 4,4 \\
\hline Tubo & 1,6 \\
\hline Aéreo & 6,1 \\
\hline Floater & 10,8 \\
\hline Lay back & 1,1 \\
\hline Snap & 3,5 \\
\hline Cut back & 2,5 \\
\hline Batida & 14,5 \\
\hline 360 & 2,1 \\
\hline Reentry & 2,1 \\
\hline Não definido/outras & 35,8 \\
\hline
\end{tabular}

da perna $(17,8 \%)$. O tronco foi atingido em 30,2\% seguido do membro superior com 19,3\%. No tronco, a região lombar foi acometida em 21,4\%, seguida pela região dorsal em $9,5 \%$. Nas distensões musculares de membro superior o braço $(10,7 \%)$ e o ombro $(9,5 \%)$ foram os mais lesados.

As lesões ligamentares somaram 6,3\% das lesões, sendo o membro inferior lesionado em $72,4 \%$; os joelhos $(51,7 \%)$ e tornozelos $(17,2 \%)$ foram os mais freqüentemente atingidos.

As fraturas (2,4\%) e luxações (3,2\%) representaram uma pequena proporção das lesões em nosso estudo. As fraturas atingiram o membro inferior em $56,5 \%$, sendo o joelho o mais lesionado (20\%), enquanto as luxações atingiram principalmente o ombro em $33 \%$.

Finalmente, as queimaduras por água-viva foram responsáveis por 8,95\% das ocorrências, atingindo principalmente o membro superior, sendo o braço acometido em 36,1\% dos casos.

A colisão com a prancha foi relatada como causa de acidente em $35,9 \%$; as quilhas $(55,2 \%)$ e o bico $(11,9 \%)$ foram as partes da prancha mais envolvidas (tabelas 5, 6 e 7). Já as manobras no surfe foram responsáveis por 35,1\% das lesões, sendo o tubo (16\%), o drop (15,5\%), a batida $(14,5 \%)$ e o floater $(10,8 \%)$ as manobras mais freqüentes envolvidas nos acidentes. Outras causas de lesões foram relatadas em $28,8 \%$.

Em mais da metade das lesões $(54,2 \%)$ o tempo de afastamento da prática do surfe foi de até 7 dias, enquanto em $20,7 \%$ foi de 7 a 14 dias (tabela 8 ). Isto nos permite admitir a baixa gravidade das lesões envolvidas com o surfe. Apenas 14,8\% das lesões mantiveram o praticante afastado por mais de 30 dias. Dos acidentados, cerca de $42,3 \%$

\begin{tabular}{|c|c|}
\hline \multicolumn{2}{|c|}{$\begin{array}{c}\text { TABELA } 8 \\
\text { Tempo de afastamento do surfe }\end{array}$} \\
\hline Afastamento & $\%$ \\
\hline Menos de 7 dias & $54,2 \%$ \\
\hline De 7 a 14 dias & $20,8 \%$ \\
\hline De 14 a 30 dias & $10,2 \%$ \\
\hline Mais de 30 dias & $14,8 \%$ \\
\hline \multicolumn{2}{|c|}{ TABELA 9} \\
\hline Tamanho das ondas, & das lesões \\
\hline Ondas & $\%$ \\
\hline Menor que $1 \mathrm{~m}$ & 29,2 \\
\hline 1 a $2 m$ & 56,1 \\
\hline Maior que $2 \mathrm{~m}$ & 9,1 \\
\hline Ressaca & 5,6 \\
\hline
\end{tabular}




\begin{tabular}{cc}
\hline \multicolumn{2}{c}{ TABELA 10} \\
Época do ano em que aconteceu o acidente \\
\hline Estação do ano \\
Primavera & $\%$ \\
Verão & 16,1 \\
Outono & 39,4 \\
Inverno & 17,6 \\
\end{tabular}

procuraram atendimento médico imediato, sendo que $64,1 \%$ deles o fizeram até o $14^{\circ}$ dia após o acidente e 35,7\% não foram ao médico após a lesão.

As lesões ligamentares (28\%), as fraturas (42,8\%), as luxações $(27,5 \%)$ e as hérnias de disco foram responsáveis pelo tempo de afastamento mais prolongado (acima de 30 dias).

Dadas as características do litoral brasileiro, onde são freqüentes ondulações de pequeno a médio porte e temperaturas elevadas, os acidentes predominaram em ondas de até $2 \mathrm{~m}(75,2 \%)$ (tabela 9 ).

Mais da metade dos acidentes aconteceram durante a primavera e o verão $(54,4 \%)$, em águas de boa temperatura $(58,4 \%)$. Apesar disso, o fato de nossa amostra ter predomínio de praticantes do Sul e Sudeste do Brasil, onde as temperaturas oceânicas são inferiores àquelas encontradas no Nordeste, um número significativo de acidentes (44,4\% das lesões) ocorreu durante o outono e inverno com temperaturas oceânicas consideradas frias ou muito frias. Em grande parte desses acidentes (águas frias) os praticantes usavam roupa de borracha (tabelas 10 e 11).

A maior parte dos acidentes ocorreu em águas com profundidade até 1 metro (47\%), sendo as entorses ligamentares e as fraturas mais freqüentes em ondas de 0,5 a 1 metro. Já os cortes, contusões, distensões musculares e as queimaduras por água-viva aconteceram em profundidades maiores que 1 metro (64,5\%); 89,6\% dos acidentes ocorreram em fundo oceânico de areia, devido a seu predomínio nas áreas de surfe da costa atlântica brasileira (tabela 12).

\section{DISCUSSÃO}

Este estudo, pioneiro no Brasil, incorpora objetivos da Organização Mundial de Saúde ${ }^{5}$ visando a redução da incidência e da gravidade das lesões esportivas antes do ano 2000, através da obtenção de dados referentes ao perfil epidemiológico das lesões do surfe e no Brasil e da proposição de medidas preventivas.

O surfe, um esporte aquático, com características de demanda aeróbia e anaeróbia de moderada a alta intensidade, enquadra-se na categoria de esporte com limitado contato físico e moderado risco de colisão, que requer elevado ní-

\begin{tabular}{|c|c|}
\hline \multicolumn{2}{|c|}{ TABELA 11} \\
\hline Temperatura & $\%$ \\
\hline Muito fria & 12,31 \\
\hline Eria & 29,28 \\
\hline Normal & 58,39 \\
\hline \multicolumn{2}{|c|}{ TABELA 12} \\
\hline \multicolumn{2}{|c|}{ Profundidade do mar durante o acidente } \\
\hline Profundidade & $\%$ \\
\hline Menor que $0,5 \mathrm{~m}$ & 11,0 \\
\hline 0,5 a $1 \mathrm{~m}$ & 36,0 \\
\hline 1 a $2 \mathrm{~m}$ & 37,2 \\
\hline Maior que $2 \mathrm{~m}$ & 15,8 \\
\hline
\end{tabular}

vel de habilidade neuromuscular e envolve movimentos dos membros superiores, inferiores e da coluna vertebral ${ }^{6}$. A dramática modificação no design e material das pranchas de surfe nos últimos 20 anos tornou-as pequenas e leves, permitindo ao praticante maior aceleração, manobrabilidade e radicalidade nos movimentos. Felizmente, a crescente popularidade do esporte, associada às modificações no design das pranchas e repertório de manobras dos praticantes, não levaram a um aumento na incidência de lesões relacionadas ao surfe. Com uma média de prática de 2,6 dias/semana, 2,1 sessões de surfe por dia, com duração de 2,68 horas, a incidência de lesões em nosso estudo (que requereram atenção médica ou que impediram a prática do surfe por um ou mais dias, num período de três anos) foi de 2,47 lesões para cada 1.000 dias de surfe. A investigação de Lowdon et al. ${ }^{2}$, uma das poucas publicações onde a comparação com nossos resultados é possível, apresentou uma incidência de 3,5 lesões para cada 1.000 dias de surfe.

Dadas as características nacionais deste estudo, foi impossível caracterizar a população em risco, considerada por definição o denominador utilizado no cálculo da incidência de lesões esportivas. Isto inviabilizou as comparações deste resultado (incidência de lesões) com estudos envolvendo outras modalidades esportivas ${ }^{7,8}$. Apesar disso, a análise epidemiológica transversal de nosso estudo permite inúmeras reflexões e considerações acerca das lesões envolvendo o surfe no Brasil.

O fato de $27 \%$ dos questionários terem sido respondidos por praticantes que não sofreram lesão reduz a possibilidade de as respostas só terem sido dadas por aqueles que tenham sofrido lesão. A maior concentração de acidentes se deu durante o free surf(surfe recreacional) e se deve, muito provavelmente, às características desta amostra, com-

. 
posta por uma maioria de praticantes recreacionais e experientes ensaiando manobras velozes e por vezes perigosas. Esses dados são consistentes com os encontrados por Lowdon et al. ${ }^{2}$ e diferem dos achados das lesões decorrentes da prática do snowboard e roller skate, em que significante parte dos acidentes envolve praticantes inexperientes ${ }^{9,10}$.

Entretanto, são necessários estudos comparativos, tendo como população-alvo um maior número de atletas amadores e profissionais em fase competitiva, já que o elevado nível de participação e a necessidade de manobras de alta performance podem tornar os acidentes mais freqüentes.

Cerca de $54 \%$ da amostra praticam o surfe entre 10 e 15 horas, estando, portanto, mais expostos aos efeitos negativos do sol. As medidas preventivas devem incentivar e reforçar a importância do uso do protetor solar, das camisas de lycra, evitando ainda o surfe nas horas de maior exposição ultravioleta.

O número superior de partes do corpo lesado (927) em relação o número de acidentados (670) se justifica pelo fato de que mais de uma parte do todo ter sido afetada no mesmo acidente. A maioria das lacerações e contusões continua a ser causada por queda da prancha com contato com as quilhas, o bico, a rabeta. As campanhas preventivas devem continuar a enfatizar a necessidade do uso do protetor de borracha para nariz (nose guard), a utilização de rabetas menos pontiagudas e as quilhas feitas com material emborrachado. Felizmente, as lesões que atingem a cabeça aparecem em menor número $(15,5 \%)$ do que foi encontrado por Lowdon et al. ${ }^{2}$ (29\%). Entretanto, pelo potencial risco de fatalidade que as contusões de crânio apresentam, medidas preventivas devem ser reforçadas entre os jovens surfistas, no sentido de protegerem-se da prancha nas quedas, devendo ser considerado o uso do capacete e do protetor de bico de prancha ${ }^{11}$.

As distensões musculares na região lombar (21,4\% do total) estão, provavelmente, relacionadas com os movimentos intensos e explosivos de rotação e compressão da coluna lombar durante o surfe. Já as lesões ligamentares, que representam $6,3 \%$, atingem joelhos $(51,7 \%)$ e tornozelos $(17,2 \%)$ como decorrência de manobras como o tubo, o drop, a batida e o floater.

Essas lesões musculoligamentares podem estar relacionadas também com a alta prevalência de recorrentes dores lombares $(28,4 \%)$, dores no pescoço $(27,3 \%)$, dores nos ombros $(20,5 \%)$ e dores nos joelhos (12,5\%). Isso sugere que as lesões de esforço repetido sejam um problema comum entre os surfistas e que possam predispor a lesões musculoligamentares de natureza traumática. Esta alta prevalência de dores recorrentes talvez possa ser atribuída às características intrínsecas do esporte, além da falta de pre- paração muscular específica por parte do surfista, sendo necessárias pesquisas mais aprofundadas nesta área.

Das características biomecânicas do surfe e que podem estar associadas à ocorrência de lesões, podem-se citar:

1) movimentos repetidos de remada - podem sobrecarregar o complexo musculotendíneo do ombro, especialmente em pranchas com baixa flutuação;

2) posição de hiperextensão isométrica da coluna dorsal e cervical durante a remada - leva ao desequilíbrio muscular;

3) movimentos repetidos de compressão e rotação dos discos intervertebrais na coluna lombar e dorsal durante as diversas manobras - podem estar envolvidos com o processo de degeneração discal;

4) posição freqüente dos joelhos em flexão e valgo pode sobrecarregar os componentes mediais do joelho.

Assim, medidas preventivas específicas devem ser desenvolvidas junto aos praticantes e devem incluir um programa de alongamento e fortalecimento musculotendíneo geral e específico, focando a região cervical, dorsal, lombar, abdominal, ombros e membros inferiores.

Deve-se considerar também, nos futuros estudos epidemiológicos sobre lesões no surfe, uma definição de lesão esportiva mais abrangente, como a proposta por Watson ${ }^{12}$, como sendo aquela que dificulta o treinamento e a competição sem causar total incapacidade.

A reduzida incidência de fraturas $(2,4 \%)$ neste estudo difere dos valores encontrados por Lowdon et al. ${ }^{2}$ (15\%). Apesar do impacto com a água ser um fator de proteção contra as fraturas, a prática do surfe em águas rasas foi responsável por dois episódios de fratura cervical, necessitando fixação interna, sendo que uma delas resultou em tetraplegia. Dentre as manobras mais comumente relacionadas às fraturas e lesões ligamentares sérias do tornozelo estão o floater, em sua fase de aterrissagem. Assim, as medidas preventivas devem incluir campanhas educacionais visando a conscientização sobre os perigos de cair da prancha e colidir com bancos de areia rasos, além de orientações sobre o transporte adequado em caso de traumas de crânio e cervical ${ }^{13}$.

Apesar da baixa incidência de hérnia de disco neste estudo, a queixa freqüente de dores lombares recorrentes em nossa amostra nos leva a supor que sua incidência pode estar pouco diagnosticada.

A elevada incidência de queimaduras por água-viva não foi encontrada no trabalho de Lowdon et al. ${ }^{3}$, provavelmente por ter sido realizado com população de praticantes do Sudeste australiano, onde a prevalência de água-viva é praticamente inexistente em águas frias. 
As perfurações de tímpano (apenas cinco casos) aconteceram devido ao trauma direto da membrana timpânica com a água durante a queda do praticante da prancha.

A maioria das lesões em nosso estudo ocorreu em fundo oceânico de areia (89,6\%), predominante em nosso litoral, em águas de boa temperatura $(58,9 \%)$ durante a primavera e outono.

A relativamente baixa incidência de lesões, somada ao fato de que em mais da metade delas (54\%) o tempo de afastamento não passou de sete dias, demonstra o baixo risco de incapacidade associado ao surfe. As fraturas $(42,8 \%)$, as luxações $(27,5 \%)$ e distensões ligamentares (28\%) foram responsáveis pelos maiores períodos de afastamento (mais de 30 dias).

\section{RECOMENDAÇÕES}

Como para a maioria dos esportes no Brasil, no surfe também não existe um sistema de coleta de dados e informações sobre os acidentes. Para o devido controle e monitorização das lesões esportivas mais graves relacionadas à

\section{REFERÊNCIAS}

1. US Consumer Product Safety Commission. Tabulation of Data from the National Eletronic Injury Surveillance System (NEISS). Washington, DC, 1979.

2. Lowdon BJ, Pitman J, Pateman NA, Ross K. Injuries to international competitive surf board riders. Sports Med Phys Fitness 1987;22:5763.

3. Lowdon BJ, Pateman NA, Pitman J. Surfboard riding injuries. Med J Aust 1983;2:613-6.

4. Hoy K, Lindblad BE, Terkelsen CJ, Hellehand HE. Handball injuries. Am J Sports Med 1992;20:441-4.

5. Backx F, Beijer H, Bol E, Erich W. Injuries in high risk persons and high risk sports. Am J Sports Med 1991;19:124-30.

6. Goldberg B. Sports and exercise for children with chronic health condition. Human Kinetics, 1995:53-9.

7. De Loes M. Epidemiology of sports injuries in the Swiss organization "Youth and Sports" 1987-1989. Int J Sports Med 1995;16:134-8.

8. Schootman M, Powell JW, Torner JC. Study designs and potential biases in sports injury research. The case control study. Sports Med 1994; 18:22-37. prática do surfe é aconselhável realizar estudos visando a implantação de um sistema nacional de controle das lesões esportivas, que contemple: 1) estimar quantitativamente a incidência de lesões para cada esporte (morbidade e mortalidade); 2) detectar os eventos esportivos com maior potencial para acidentes; 3 ) identificar as circunstâncias relacionadas ao acidente; 4) estimular as pesquisas epidemiológicas para determinar fatores específicos para o controle e a prevenção; 5) determinar a eficácia dos programas de prevenção ${ }^{14}$.

Apesar da baixa incidência de lesões esportivas, com o crescente número de praticantes de surfe cada vez mais jovens, atraídos por fatores socioculturais e pela criação de inúmeras "escolas de surfe", além da introdução do surfe como modalidade esportiva em aulas de educação física escolar, as campanhas preventivas visando reduzir as lesões traumáticas e de overuse devem ser iniciadas e introduzidas nas escolas ${ }^{15}$ e divulgadas pela mídia específica.

Projeto financiado pelo $\mathrm{CNPq}$

9. Abu-Laban RB. Snowboarding injuries: an analysis and comparison with alpine skiing injuries. Can Med Assoc J 1991;145:1097-103.

10. Muller LD, Renstrom PHF, Pyne JIB. Injuries in skating. Clinical practice of sports injuries. Prevention and care. Blackwell, 1994, 711-5.

11. Lowdon BJ. Surfing injuries: immediate and long term problems and prevention. Athletic Training 1984;19:105-8.

12. Watson AWS. Incidence and nature of sports injuries in Ireland. Am J Sports Med 1993;21

13. Steinman J. As lesões e os acidentes mais comuns no surf. Revista Inside 1995;100, 26.

14. Laporte RE, Kohl WH, Dearwater SR, Kriska AM, Anderson R, Aaron DJ, Olsen T, Mccarty DJ. Surveillance of serious recreational injuries: a capture-recapture approach. Med Sci Sports Exerc 1992;25:204-9.

15. Current comment from the American College of Sports Medicine. The prevention of sports injuries of children and adolescent. Med Sci Sports Exerc 1993;25(suppl 8):1-7. 